\title{
Spectra and binding energy predictions of chiral interactions for ${ }^{7} \mathrm{Li}$
}

\author{
A. Nogga, ${ }^{1}$ P. Navrátil,${ }^{2}$ B.R. Barrett,${ }^{3}$ and J.P. Vary ${ }^{4}$ \\ ${ }^{1}$ Institut für Kernphysik, Forschungszentrum Jülich, 52425 Jülich, Germany* \\ ${ }^{2}$ Lawrence Livermore National Laboratory, L-14, \\ P.O. Box 808, Livermore, California 94551, USA \\ ${ }^{3}$ Department of Physics, University of Arizona, Tucson, Arizona 85721, USA 7 \\ ${ }^{4}$ Department of Physics and Astronomy, Iowa State University, Ames, Iowa 50011, USA
}

(Dated: November 28, 2005)

\begin{abstract}
Using the no-core shell model approach, we report on the first results for ${ }^{7} \mathrm{Li}$ based on the next-tonext-to-leading order chiral nuclear interaction. Both, two-nucleon and three-nucleon interactions are taken into account. We show that the $p$-shell nuclei are sensitive to the subleading parts of the chiral interactions including three-nucleon forces. Though chiral interactions are soft, we do not observe overbinding for this $p$-shell nucleus and find a realistic description for the binding energy, excitation spectrum and radius.
\end{abstract}

PACS numbers: PACS number(s): 21.10.-k,21.30.-x,27.20.+n,21.60.-n

\section{INTRODUCTION}

We are currently experiencing rapid progress in our understanding of nuclear properties. This is triggered by two major developments.

On one hand, we have increasingly powerful supercomputers, for which new and very efficient algorithms have been developed to solve the nuclear many-body problem. We are now able to solve the Schrödinger equation for realistic nuclear interactions for $p$-shell nuclei including also three-nucleon forces (3NF's) 1, 2]. This is a major advance in itself, because it is becoming more clear that reliable predictions for many nuclear observables, binding energy and spectra can be obtained from phenomenological nuclear two- $(\mathrm{NN})$ and three-nucleon $(3 \mathrm{~N})$ interaction models. Especially, for the NN system, these have reached a high degree of sophistication and describe the NN data up to pion production threshold perfectly 3, 4, 5, 6]. For the binding energies of $p$-shell nuclei, the structure of the 3NF's turned out to be significant, leading to improved models of these forces engineered to describe a wide range of light nuclei accurately [7]. This tool is of increasing importance to determine, e.g. reaction rates for astrophysical processes [8], which are experimentally not accessible or properties of nuclei with large neutron excess [1].

On the other hand, there was a great deal of progress in our understanding of how chiral perturbation theory $(\mathrm{ChPT})$ can be extended from purely pionic or the nucleon-pion system (for a review see e.g. 9]) to systems with more than one nucleon [10, 11, 12]. In this approach, one makes use of the explicit and spontaneous breaking of chiral symmetry to systematically expand the

\footnotetext{
*Electronic address: a.nogga@fz-juelich.de

${ }^{\dagger}$ Electronic address: navratil1@llnl.gov

${ }^{\ddagger}$ Electronic address: bbarrett@physics.arizona.edu

$\S$ Electronic address: jvary@iastate.edu
}

strong interaction in terms of a generic small momentum. Thereby, the NN interactions, the 3NF's and also $\pi \mathrm{N}$ scattering are related to each other. The chiral symmetry and the pattern of its breaking are not systematically taken into account by today's phenomenological interactions, except that in all of them the longest range part of the potential is generally the one pion exchange interaction. Therefore, though QCD is believed to be the theory of the strong interaction for the energies of relevance in nuclear systems, we are not able to perform confirming tests using the traditional forces. This will be possible using nuclear forces based on ChPT. It will be especially important to look at subleading parts of the interaction, which include the $2 \pi$ exchange $\mathrm{NN}$ and $3 \mathrm{~N}$ forces. Here, many of the relations between $\mathrm{NN}, 3 \mathrm{~N}$ and $\pi \mathrm{N}$ interactions become apparent. Therefore, finding signatures of the 3NF's is an important aspect of current research on this issue.

In the past, the effects of 3NF's have been studied using the phenomenological models. In nucleon-deuteron $(\mathrm{Nd})$ scattering above $60-100 \mathrm{MeV}$ lab energy, it was found that predictions for some (polarization) observables depend on the 3NF model used, but not on the NN interaction chosen [13]. These observables are excellent candidates to pin down the structure of 3NF's and, therefore, outstanding laboratories to study the important subleading parts of the chiral interactions. Consequently, a lot of experiments were triggered, which provide an important set of data to probe these models [14, 15, 16, 17]. First comparisons for chiral interactions to the data were performed and, in general, agreement was found in the energy range, where chiral interactions are expected to work 18.

These data, however, are manifestly isospin $T=1 / 2$ and, therefore, not suitable to probe the $3 \mathrm{NF}$ in the isospin $T=3 / 2$ channel. But it was argued that the $T=3 / 2$ component is very important to describe $p$-shell nuclei, which mainly motivated the new $3 \mathrm{NF}$ terms in the Illinois series of $3 \mathrm{~N}$ interactions [7]. This clearly shows that the spectra and binding energies of light nuclei are 
complementary to nd scattering and provide indispensable information on the structure of 3NF's. It is therefore of utmost importance to predict properties of $p$-shell nuclei based on the chiral interactions including these 3NF's.

Chiral interactions are low momentum interactions. The unknown short distance part of the force is absorbed in a tower of contact terms. Necessarily, we need to regularize the interactions with cutoff functions. It turns out that one obtains a decent description of the NN data using rather small cutoffs. This is advantageous, because the resulting interactions are soft and convergence is generally faster than the convergence with traditional models. But this has also been criticized in the past. The experience with traditional models indicated a need for rather hard cores not only in the $\mathrm{NN}$ interaction, but also in the $3 \mathrm{NF}$ to prevent strong overbinding in systems beyond the $s$-shell. This is a surprising result, because it means that we cannot properly separate high energy degrees of freedom from the low energy part. Because the high momentum tail of traditional interactions is strongly model dependent, a sensitivity to this tail will induce a strong model dependence of the results. Indeed, the binding energies of nuclei do depend on the chosen $\mathrm{NN}$ interaction model. It is believed that this model dependence is strongly reduced, once 3NF's consistent with the NN force are added (in fact it was proven that any NN interaction can be augmented by a $3 \mathrm{NF}$ so that the description of the $3 \mathrm{~N}$ data is identical to that of a second phase-equivalent NN force [19], thereby removing the model dependence). Similarly, one can expect that a consistent combination of $\mathrm{NN}$ and $3 \mathrm{~N}$ force will lead to a reasonable description of light nuclei even if the high momentum tail is completely missing. An important aspect of this work will be to confirm this expectation by an explicit calculation.

Because of the strong non-locality of today's chiral interactions, to the best of our knowledge, there is only one reliable many-body technique to solve the Schrödinger equation for $p$-shell nuclei for these interactions including the $3 \mathrm{NF}^{\prime}$. . This is the ab-initio no-core shell model (NCSM) 2, 20, 21]. In this paper, we use the NCSM to predict spectra and binding energies for ${ }^{7} \mathrm{Li}$. These results will be presented along with calculations for the $s$-shell states for ${ }^{3} \mathrm{H}$ and ${ }^{4} \mathrm{He}$, which were used to determine unknown parameters of the $3 \mathrm{NF}$. The $p$-shell results are then predictions.

The manuscript is organized as follows. In Section II we start with a brief summary of the status on the research on chiral interaction models, mainly to explain the force model entering in the calculations. The NCSM approach is introduced in Section III to keep this paper self-contained. Then, in Section IV we discuss our numerical results. Conclusions and an outlook will be given in Section $\nabla$

\section{CHIRAL INTERACTIONS}

Microscopic nuclear structure is generally based on NN interactions, which describe the NN scattering data up to $\pi$-production threshold perfectly. Usually, such interactions are based on the $1 \pi$-exchange, which is augmented by a more or less phenomenological short range part. It turns out that binding energies based on these interactions are model dependent and too small [1, 22, 23]. $3 \mathrm{NF}$ 's are clearly necessary.

However, defining consistent combinations of NN and $3 \mathrm{~N}$ forces is not a trivial task, and rarely are both based on a single underlying theory. In practice, the NN interaction models are usually augmented by a $2 \pi$ exchange 3NF 24, 25] or extended versions thereof 7]. Then parameters are mostly adjusted to reproduce the binding energies of $s$-shell nuclei or nuclear matter density. Though this can be very successful in the description of nuclear properties 7], it lacks a solid theoretical foundation. The relation to QCD is completely lost. Processes of other strongly interacting systems, like the $\pi \mathrm{N}$ system, are not quantitatively related to these forces, though the basic mechanisms involve these particles.

Since lattice simulations for nuclei are not realistic for the foreseeable future, effective field theory is an appealing theoretical foundation on which to build. In its simplest form, an effective field theory was formulated, that explicitly takes only nucleons into account. It is accompanied by a power counting in terms of powers of $r / a$, where $r$ is the effective range of the interaction and $a$ the large scattering length of the NN system (for a recent review see 26$]$ ). This property is realized in very different physical systems, e.g. ${ }^{4} \mathrm{He}$ clusters and atoms close to a Feshbach resonance. It allows one to identify universal properties. Also, it is possible to identify nuclear properties, which are dominated by the large scattering length of the NN interaction. An example is the correlation between ${ }^{3} \mathrm{H}$ and ${ }^{4} \mathrm{He}$ binding energies, known as Tjonline, which is naturally explained using this approach [27. While these results are interesting in themselves, they are by construction not that interesting for the relation of QCD and nuclear physics. The approach will only describe observables, which do not reflect any specific property of QCD except the large scattering length.

To sense the leading role of QCD, another effective field theory is the tool of choice: ChPT. The new physical input is the approximate chiral symmetry of QCD, which is known to be spontaneously broken. Based on this an effective field theory can be formulated that involves nucleons and $\pi$ 's explicitly. The Goldstone-boson character of the $\pi$ 's (related to the spontaneous breaking of chiral symmetry) guarantees that amplitudes in purely pionic and the $\pi \mathrm{N}$ system can be expanded in powers of a small, typical momentum $Q$. The explicit breaking of chiral symmetry due to the finite quark masses is expanded similarly in terms of the $\pi$ mass [28]. Electromagnetic effects on the quark level and quark mass differences have also been taken into account by an ex- 
pansion of isospin symmetry breaking contributions in terms of the fine structure constant and the quark mass differences [29, 30].

Obviously, the NN system is different. The deuteron bound state clearly shows that a perturbative treatment is impossible. Weinberg realized that this is understood by an enhancement of reducible diagrams in two- or more-nucleon systems and proposed to expand the irreducible diagrams perturbatively in terms of small external momenta, but then sum all these diagrams to infinite order using a Lippmann-Schwinger or Schrödinger equation [31, 32]. In other words, Weinberg suggested to obtain nuclear potentials from ChPT. This naturally explains that $\mathrm{NN}$ interactions are much more important than 3NF's and that these are more relevant than higherbody interactions 33. The approach was quantitatively confirmed 34, 35, 36. For the NN system, terms up to order momentum $Q^{4}$ have been taken into account nowadays 37,38 and lead to a description of $\mathrm{NN}$ data, which is comparable to the one of the phenomenological interactions. The approach to sum all irreducible diagrams to infinite order is usually called "Weinberg counting". Other schemes to deal with the non-perturbativity have been proposed [39] and are formally more consistent, because the renormalization is understood analytically. But the formal problems of the "Weinberg" scheme, which were pointed out in [39], can be circumvented by small cutoffs of the order of $500 \mathrm{MeV}$ for the regularization of the Lippmann-Schwinger equation. Under this condition, "Weinberg power-counting" is numerically more successful than the scheme of [39] as was shown in [40]. Indirect confirmation of the Weinberg approach is the favorable agreement of parameters extracted from $\pi \mathrm{N}$ scattering [4] and an NN phase shift analysis 42, 43]. Therefore, for the rest of this paper, we will rely on the Idaho-N3LO interaction [37] based on "Weinberg counting" for the NN interaction. It takes terms up to order $Q^{4}$ into account and is able to describe the NN scattering data below $\pi$ production threshold perfectly. We stress that this can only be a first step, because a complete analysis of the chiral interactions requires further order-by-order analysis to confirm convergence.

Very soon the leading 3NF's, which appear at order $Q^{3}$ were derived [44]. They consist of a $2 \pi$ exchange term, which is very similar to the widely used TucsonMelbourne 3NF 25] and, additionally, there are contact interactions with and without $1 \pi$ exchange. In the original paper, five such contact terms were believed to be independent. Fortunately, it turned out later, that only two of them remain independent once the identity of the nucleons is taken into account. In this simplified form the 3NF's were applied to $\mathrm{Nd}$ scattering, the ${ }^{3} \mathrm{H}$ and ${ }^{4} \mathrm{He}$ bound states [18]. For completeness, the explicit expressions for these forces are given in Appendix $\mathrm{A}$

For the $2 \pi$-exchange term, the power of this systematic scheme to derive consistent $\mathrm{NN}$ and $3 \mathrm{~N}$ interactions comes into play. All vertices also occur in NN interaction diagrams. This completely determines the strength

\begin{tabular}{l|rrr} 
& $c_{1}$ & $c_{3}$ & $c_{4}$ \\
\hline Fettes et al. [45] & -1.23 & -5.94 & 3.47 \\
Büttiker et al. [41] & -0.81 & -4.70 & 3.40 \\
Rentmeester et al. [42] & -0.76 & -4.78 & 3.96 \\
Entem et al. [37] & -0.81 & -3.20 & 5.40
\end{tabular}

Table I: Various determinations of the strength constants $c_{i}$ of the $3 \mathrm{NF}$. All values are in $\mathrm{GeV}^{-1}$.

and form of this part of the 3NF. As outlined in the appendix, there are three less well known strength constants in this part of the $3 \mathrm{NF}: c_{1}, c_{3}$ and $c_{4}$. They determine the contribution of various spin-isospin structures to the offshell $\pi \mathrm{N}$ scattering amplitude entering the $3 \mathrm{NF}$. Table summarizes the results of recent determinations of these constants. The most fundamental determinations were done in Ref. 41, 45 using a fit to $\pi \mathrm{N}$ scattering data. For this system, ordinary ChPT without resummation is used. The possibility to extract these constants from $\pi \mathrm{N}$ data makes the strong link of the nuclear interaction and other strong interaction processes in ChPT explicit. The rather scarce set of data for the $\pi \mathrm{N}$ system induces rather large uncertainties. This has partly been circumvented in [41] using dispersion relations. Therefore, the determination of Ref. [41] is usually considered to be more accurate. Therefore, it is nice to see that this result compares very well with the determination of [42], where the $c_{i}$ 's were extracted from a partial wave analysis of NN data. For the Idaho-N3LO interaction, the authors of 37] preferred their own determination for $c_{3}$ and $c_{4}$ based on a fit to phase shifts in high partial waves, also shown in the table. It slightly deviates from the two previous values and lead to an intense debate on the correct way to extract these constants from $\mathrm{NN}$ data. We cannot contribute to this discussion, but will use the $c_{i}$ 's of 37], simply because we want consistency of the NN and $3 \mathrm{~N}$ forces. The exact magnitude of these constants should be of less importance, because they only appear in subleading terms of minimal order $Q^{3}$. However, this issue has to be kept in mind, when we compare our results to the experimental values, especially for observables, where 3NF's are important. Please note that we will combine an NN interaction of order $Q^{4}$ to a $3 \mathrm{NF}$ of order $Q^{3}$ only. Because we will not study the convergence of our predictions with respect to the chiral expansion in this work, we think that this combination is justified. It is the state-of-the-art for chiral interaction models.

For the remaining two contact 3NF's, two more strength constants enter: $c_{D}$ and $c_{E}$ (see the appendix for the definition). In Ref. [18], it was shown that the determination of these constants is possible by a fit to the ${ }^{3} \mathrm{H}$ binding energy and the $\mathrm{Nd}$ doublet scattering length. This is only one of the possibilities to determine these constants. For the Idaho-N3LO force, we therefore decided to fit to the ${ }^{3} \mathrm{H}$ and ${ }^{4} \mathrm{He}$ binding energies. For our study here, this has two advantages. Firstly, a very accurate fit is possible, because both binding energies are well 


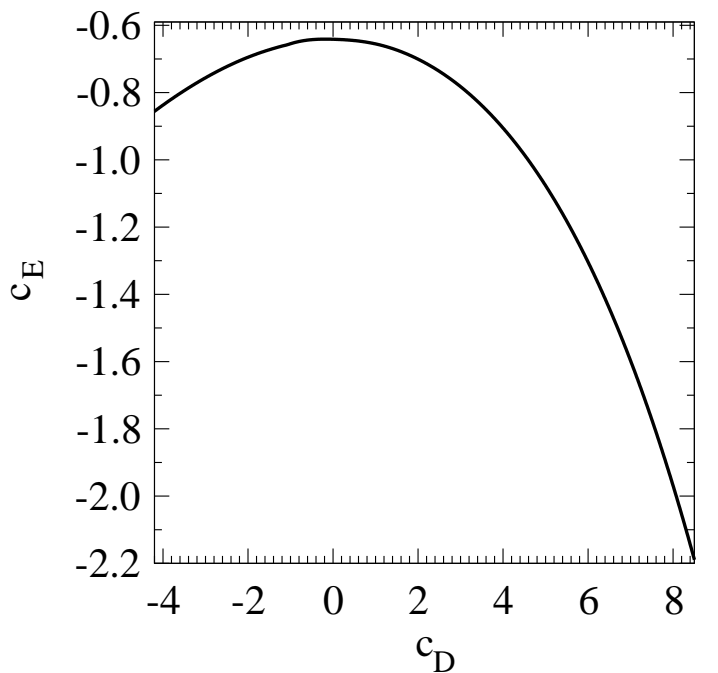

Figure 1: Correlation of the two dimensionless strength constants $c_{D}$ and $c_{E}$ of the $3 \mathrm{NF}$. All combinations of $c_{D}$ and $c_{E}$ describe the ${ }^{3} \mathrm{H}$ binding energy equally well.

\begin{tabular}{l|rr} 
& $c_{D}$ & $c_{E}$ \\
\hline 3NF-A & -1.11 & -0.66 \\
3NF-B & 8.14 & -2.02
\end{tabular}

Table II: Combinations of $c_{D}$ and $c_{E}$, which describe the ${ }^{3} \mathrm{H}$ and ${ }^{4} \mathrm{He}$ binding energies.

known experimentally. Secondly, it makes a comparison of our results to calculations with the phenomenlogical model AV18/Urbana-IX more meaningful, because this combination describes both binding energies reasonably well, but fails for some $p$-shell spectra. The fit was performed using Faddeev-Yakubovsky calculations. For a detailed description of the technique, we refer to 22. Using the ${ }^{3} \mathrm{H}$ binding energy, we established a relation between $c_{D}$ and $c_{E}$ shown in Fig. 1 For all these combinations of $c_{D}$ and $c_{E}$, Idaho N3LO in combination with the $3 \mathrm{NF}$ predict a ${ }^{3} \mathrm{H}$ binding energy of $8.482 \mathrm{MeV}$ in agreement with experiment. These combinations then enter in a calculation of the Yakubovsky eigenvalue $\eta$ for ${ }^{4} \mathrm{He}$ using the $\alpha$ particle binding energy of $28.3 \mathrm{MeV}$ as trial energy. Our theoretical prediction agrees with $28.3 \mathrm{MeV}$, if $\eta$ is equal to one. From the results shown in Fig. 2] we can read off that two combinations of $c_{D}$ and $c_{E}$ describe ${ }^{3} \mathrm{H}$ and ${ }^{4} \mathrm{He}$ equally well. For later reference, we call them "3NF-A" and "3NF-B". The numerical values are listed in Table II

We did not perform complete calculations for the binding energy for ${ }^{4} \mathrm{He}$ for each combination of $c_{D}$ and $c_{E}$. However, generally, one can expect from the ranges of $\eta$ results that the binding energy would change by approximately $2 \mathrm{MeV}$ in the range of $c_{D}$ 's considered. This sets a scale for the $Q^{3}$ contribution to ${ }^{4} \mathrm{He}$. For completeness, we also note that the pp force was augmented by the Coulomb interaction for point like protons. No

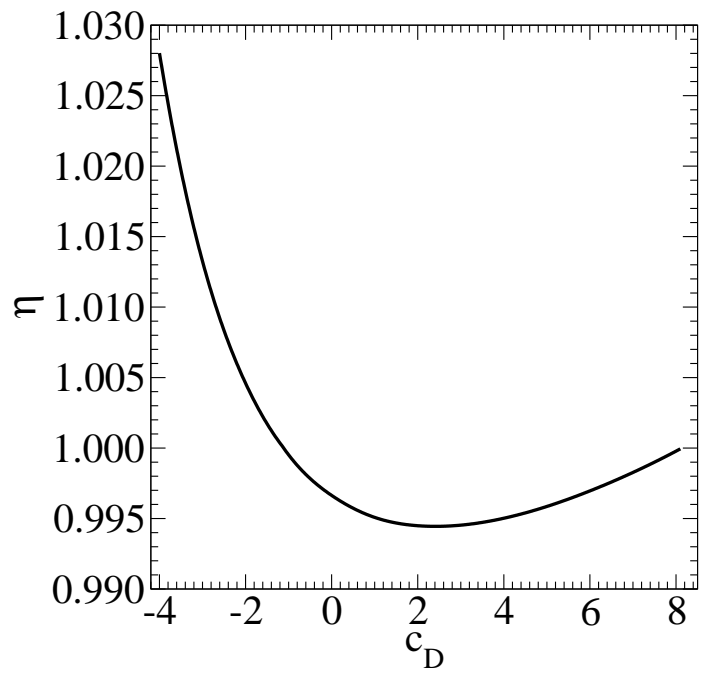

Figure 2: Eigenvalues $\eta$ of the Yakubovsky equations for ${ }^{4} \mathrm{He}$ depending on $c_{D}$. $c_{E}$ is chosen according to Fig. 1 $\eta=1$ corresponds to describing the ${ }^{4} \mathrm{He}$ binding energy exactly.

further electromagnetic contribution was taken into account as they are expected to be of less importance. We also do not include the first relativistic corrections to the $3 \mathrm{NF}$, which some people do count as an order $Q^{3}$ contribution. We cannot discuss this issue here. We only claim that, whatever counting you prefer, the relativistic corrections will be smaller than our $Q^{3}$, because we use cutoffs around $500 \mathrm{MeV}$, which are much smaller than the nucleon mass. Then, because no four-nucleon forces enter at order $Q^{3}$, our interaction is complete up to this order. Now we need to extend our calculations to the $p$-shell. For this, we will give a brief introduction to the NCSM in the next section.

\section{NCSM APPROACH}

Usually shell-model calculations exploit the possibility to assume an inert nuclear core. Taking only the valence nucleons as active particles clearly has the advantage to keep the number of many-nucleon states manageable, but, so far, it also means that effective shell model interactions have to be used, which cannot be related directly to nuclear interactions, as they have been developed for few-nucleon systems. The "ab-initio no-core shell model (NCSM)" or, more simply, the NCSM is different [20, 21]. Because all nucleons are taken to be active, in principle, the same interactions can be used for the NCSM as for traditional few-body calculations. However, for realistic nuclear forces, the wave function is hardly described by combinations of single particle states. Using harmonic oscillator (HO) states, which allow for an easy separation of the center of mass (CM) motion from the internal degrees of freedom, one encounters the additional problem to describe the the exponential long range tail of the wave function. Again effective interactions become 
necessary to achieve convergence, with a small number of HO states taken into account. These effective interactions are systematically related to the "bare" interactions [46, 47].

The scheme described in Ref. 47] is based on unitary transformations of the Hamiltonian [48], which decouples the model space from the complete Hilbert space describing the quantum mechanical system. Such transformations have already proven to be useful in a wide variety of nuclear physics problems. They allow us to decouple parts of the Fock space, such as the two nucleon space, from the rest of the Fock space [4]. Also a decoupling of low and high momentum components has been performed using the same approach [50, 51]. In many-body problems, it has not only been used for shell model calculations, but, recently, could be exploited for the solution of nuclear problems in the hyperspherical harmonics basis [52, 53].

The starting point of all NCSM calculations is a nonrelativistic $A$-body Hamiltonian, which includes NN and, important here, $3 \mathrm{~N}$ interactions

$$
H_{A}=\sum_{i=1}^{A} \frac{{\overrightarrow{p_{i}}}^{2}}{2 m_{i}}+\sum_{i<j=1}^{A} V_{i j}+\sum_{i<j<k=1}^{A} V_{i j k}
$$

$\vec{p}_{i}$ are the momenta of the $A$ particles with masses $m_{i}$. In the following, we assume that the interaction does not change the type of particle and that the total mass $M_{A}=$ $\sum_{i} m_{i}$ is well defined. Adding the CM HO potential, one can rewrite the Hamiltonian as 54

$$
\begin{aligned}
H_{A}^{\Omega}= & H_{A}+\frac{M_{A} \Omega^{2}}{2} \vec{R}^{2}=\sum_{i=1}^{A}\left(\frac{{\overrightarrow{p_{i}}}^{2}}{2 m_{i}}+\frac{m_{i} \Omega^{2}}{2}{\overrightarrow{r_{i}}}^{2}\right) \\
& +\sum_{i<j=1}^{A}\left(V_{i j}-\frac{m_{i} m_{j}}{2 M_{A}} \Omega^{2}\left(\vec{r}_{i}-\vec{r}_{j}\right)^{2}\right) \\
& +\sum_{i<j<k=1}^{A} V_{i j k}
\end{aligned}
$$

with the CM position $\vec{R}=\frac{1}{M_{A}} \sum_{i} m_{i} \vec{r}_{i}$.

In the following we would like to establish an unitary tranformation of the Hamiltonian, which decouples two parts of the Hilbert space. A rather small finite model space $P$ and the rest of the Hilbert space $Q$. The projection operators on the two spaces are also called $P$ and $Q$. They fulfill the relation $Q=1-P$.

Okubo [4] showed that the unitary operator can be derived from an operator $\omega$, which fulfills the following relations:

$$
\begin{aligned}
\omega & =Q \omega P \\
0 & =Q e^{-\omega} H_{A}^{\Omega} e^{+\omega} P \\
& =Q(1-\omega) H_{A}^{\Omega}(1+\omega) P
\end{aligned}
$$

The transformation $e^{\omega}$ already decouples the subspaces $P$ and $Q$, but it is not unitary. Unitarity can be achieved using the antihermitian operator $S=\operatorname{arctanh}\left(\omega-\omega^{\dagger}\right)$ and defining the unitary operator $U=e^{+S}$. Using the properties of $S$ and $\omega$, one finds the explicit expression 48]

$$
U=e^{S}=\left(1+\omega-\omega^{\dagger}\right)\left(1+\omega \omega^{\dagger}+\omega^{\dagger} \omega\right)^{-1 / 2}
$$

The problem is reduced to finding the operator $\omega$. For that, one defines a set of $d_{P}$ eigenstates $|n\rangle$ of the Hamilton operator $H_{A}^{\Omega}$, where $d_{P}$ is the dimension of the model space $P$. Defining $\omega|n\rangle \equiv Q|n\rangle$, it is easy to show that the decoupling condition Eq. (4) holds. This completely defines the $\omega$ operator for sets of eigenstates $|n\rangle$, for which the $P|n\rangle$ are linearly independent. In practice, for resonable choices of the eigenstates, this condition is always fulfilled. Using Eq. (3) the action of $\omega$ on $|n\rangle$ reads

$$
\begin{aligned}
\left\langle\alpha_{Q} \mid n\right\rangle & =\sum_{\alpha_{P}}\left\langle\alpha_{Q}|\omega| \alpha_{P}\right\rangle\left\langle\alpha_{P} \mid n\right\rangle \\
& \equiv \sum_{\alpha_{P}}\left\langle\alpha_{Q}|\omega| \alpha_{P}\right\rangle N_{\alpha_{P} n}
\end{aligned}
$$

$\alpha$ enumerates the basis states. The indices $P$ and $Q$ restrict enumeration to the respective subspace. With the help of the inverse $M=N^{-1}$, the matrix elements of $\omega$ are found [55] easily in a non-iterative scheme

$$
\left\langle\alpha_{Q}|\omega| \alpha_{P}\right\rangle=\sum_{n}\left\langle\alpha_{Q} \mid n\right\rangle M_{n} \alpha_{P}
$$

As elaborated in 56], there is always as set of states $|n\rangle$, for which the inverse of $N$ exists. In practice, one chooses the $d_{P}$ lowest lying eigenstates of $H_{A}^{\Omega}$. Because $\left(1-\omega+\omega^{\dagger}\right)|n\rangle$ has only $P$-space components, the same holds for $U^{\dagger}|n\rangle$. Matrix elements of states $|n\rangle$ are then exactly reproduced in the model space

$$
\left\langle n^{\prime}|O| n\right\rangle=\left\langle n^{\prime}|U \underbrace{P U^{\dagger} O U P}_{O_{\text {eff }}} U^{\dagger}| n\right\rangle
$$

Because we are only interested in the $P$-space matrix elements of $O_{\text {eff }}$, we can make use of the simplification

$$
U P=(1+\omega)\left(1+\omega^{\dagger} \omega\right)^{-1 / 2} P
$$

and write

$$
\begin{gathered}
\left\langle\alpha_{P}\left|O_{\text {eff }}\right| \beta_{P}\right\rangle=\sum_{\alpha_{P}^{\prime} \beta_{P}^{\prime}}\left\langle\alpha_{P}\left|\left(1+\omega^{\dagger} \omega\right)^{-1 / 2}\right| \alpha_{P}^{\prime}\right\rangle \\
\sum_{n n^{\prime}} M_{n \alpha_{P}^{\prime}}^{*} M_{n^{\prime} \beta_{P}^{\prime}}\left\langle n|O| n^{\prime}\right\rangle \\
\left\langle\beta_{P}^{\prime}\left|\left(1+\omega^{\dagger} \omega\right)^{-1 / 2}\right| \beta_{P}\right\rangle
\end{gathered}
$$

Here we made use of the relation

$$
\begin{aligned}
& \left\langle\alpha_{P}\left|\left(1+\omega^{\dagger}\right) O(1+\omega)\right| \beta_{P}\right\rangle \\
& =\sum_{n n^{\prime}} M_{n \alpha_{P}}^{*} M_{n^{\prime} \beta_{P}}\left\langle n|O| n^{\prime}\right\rangle
\end{aligned}
$$

There are two special cases of interest of Eq. (11). The first, $O=1$, is useful to determine the $P$ space renormalization operator $\left(1+\omega^{\dagger} \omega\right)^{-1 / 2}$ since

$$
\left\langle\alpha_{P}\left|\left(1+\omega^{\dagger}\right)(1+\omega)\right| \beta_{P}\right\rangle
$$




$$
=\left\langle\alpha_{P}\left|\left(1+\omega^{\dagger} \omega\right)\right| \beta_{P}\right\rangle=\sum_{n} M_{n \alpha_{P}}^{*} M_{n \beta_{P}}
$$

The second, $O=H_{A}^{\Omega}$, makes the evaluation of the effective Hamiltonian especially simple

$$
\begin{aligned}
\left\langle\alpha_{P}\left|\mathcal{H}_{e f f}\right| \beta_{P}\right\rangle= & \left\langle\alpha_{P}\left|\left(1+\omega^{\dagger}\right) H_{A}^{\Omega}(1+\omega)\right| \beta_{P}\right\rangle \\
& =\sum_{n} M_{n \alpha_{P}}^{*} M_{n \beta_{P}} E_{n}
\end{aligned}
$$

with $E_{n}$ the eigenenergies of the eigenstates $|n\rangle$. These equations are exact. They reduce the problem to one in a very small model space $P$. However, because solutions of the problem are necessary to obtain the effective operator, they do not help to solve the eigenvalue problem in this form.

The NCSM concept becomes useful by approximating the effective interaction in an $a$-body cluster approximation. There are two obvious constraints on this approach. Firstly, to keep the approximation controllable, we require that the effective interaction approaches the bare interaction for model spaces $P \rightarrow \infty$, and, secondly, we expect best convergence, when the nuclear part of the interaction acts in the $a$-body cluster with the same strength as in a free $a$-body system. Then, under the assumption that only $b=2$-body bare forces act, one needs to solve the cluster problem for

$$
\begin{aligned}
H_{a}^{\Omega}= & \sum_{i=1}^{a}\left(\frac{{\overrightarrow{p_{i}}}^{2}}{2 m_{i}}+\frac{m_{i} \Omega^{2}}{2}{\overrightarrow{r_{i}}}^{2}\right) \\
& +\sum_{i<j=1}^{a}\left(V_{i j}-\frac{m_{i} m_{j}}{2 M_{A}} \Omega^{2}\left(\vec{r}_{i}-\vec{r}_{j}\right)^{2}\right)
\end{aligned}
$$

Note that in this cluster Hamiltonian, the strength of the HO two-body potential depends on the original $A$. This induces a confining meanfield potential for the cluster, which simply reflects that the cluster is embedded in the nuclear environment. It has the nice property that we can base our effective interaction on an infinite set of cluster bound states. No scattering states need to be considered. The effective interaction for $b$-body interactions in the $a$-body cluster approximation for the $A$-body system is defined as

$$
\mathcal{V}_{e f f}^{A, a, b}=\mathcal{H}_{e f f}-\sum_{i=1}^{a}\left(\frac{{\overrightarrow{p_{i}}}^{2}}{2 m_{i}}+\frac{m_{i} \Omega^{2}}{2}{\overrightarrow{r_{i}}}^{2}\right)
$$

where $\mathcal{H}_{\text {eff }}$ is derived from an Hamiltonian operator like given in Eq. (14). The effective interaction enters into the $A$-body problem with a different weight, that insures that the bare interaction is recovered for large model spaces [20, 21]

$$
\begin{aligned}
\mathcal{H}_{e f f}^{A}= & \sum_{i=1}^{A}\left(\frac{{\overrightarrow{p_{i}}}^{2}}{2 m_{i}}+\frac{m_{i} \Omega^{2}}{2}{\overrightarrow{r_{i}}}^{2}\right) \\
& +\frac{(A-a) !(a-b) !}{(A-b) !} \sum_{i_{1}<i_{2} \ldots<i_{a}=1}^{A} \mathcal{V}_{e f f, i_{1} \ldots i}^{A, a, b}
\end{aligned}
$$

In this form it becomes apparent that we have a double counting problem, when both, $b=2$ and $b=3$ interactions enter the cluster Hamiltonian. This can be solved as outlined in [2] and requires two effective interactions. The first one, $\mathcal{V}_{e f f, i_{1} \ldots i_{a}}^{A, a, N N}$, is based on the Hamiltonian in Eq. (14). The second one, $\mathcal{V}_{e f f, i_{1} \ldots i_{a}}^{A, a, 3 N}$, is based on

$$
\begin{aligned}
H_{a}^{\Omega}= & \sum_{i=1}^{a}\left(\frac{{\overrightarrow{p_{i}}}^{2}}{2 m_{i}}+\frac{m_{i} \Omega^{2}}{2}{\overrightarrow{r_{i}}}^{2}\right) \\
& +\sum_{i<j=1}^{a}\left(V_{i j}-\frac{m_{i} m_{j}}{2 M_{A}} \Omega^{2}\left(\vec{r}_{i}-\vec{r}_{j}\right)^{2}\right) \\
& +\sum_{i<j<k=1}^{a} V_{i j k}
\end{aligned}
$$

which additionally takes into account the $3 \mathrm{NF}$. The combination

$$
\begin{aligned}
& \mathcal{H}_{\text {eff }}^{A}= \sum_{i=1}^{A}\left(\frac{{\overrightarrow{p_{i}}}^{2}}{2 m_{i}}+\frac{m_{i} \Omega^{2}}{2}{\overrightarrow{r_{i}}}^{2}\right) \\
&+\frac{(A-a) !(a-3) !}{(A-3) !} \sum_{i_{1}<\ldots<i_{a}=1}^{A} \mathcal{V}_{e f f, i_{1} \ldots i_{a}}^{A, a, 3 N} \\
&+\frac{(A-a) !((A-3) !(a-2) !-(A-2) !(a-3) !)}{(A-3) !(A-2) !} \\
& \sum_{i_{1}<\ldots<i_{a}=1}^{A} \mathcal{V}_{e f f, i_{1} \ldots i_{a}}^{A, a, N N}
\end{aligned}
$$

then fulfills the constraints for our effective interaction. Specifically for $a=3$, we get

$$
\begin{aligned}
\mathcal{H}_{e f f}^{A}= & \sum_{i=1}^{A}\left(\frac{{\overrightarrow{p_{i}}}^{2}}{2 m_{i}}+\frac{m_{i} \Omega^{2}}{2}{\overrightarrow{r_{i}}}^{2}\right) \\
& +\sum_{i_{1}<\ldots<i_{a}=1}^{A} \mathcal{V}_{e f f, i_{1} \ldots i_{a}}^{A, a, 3 N} \\
& +\frac{3-A}{A-2} \sum_{i_{1}<\ldots<i_{a}=1}^{A} \mathcal{V}_{e f f, i_{1} \ldots i_{a}}^{A, a, N N}
\end{aligned}
$$

This interaction will be used in the calculations in the next section. It will enhance the convergence so that we can obtain meaningful binding energies for $p$-shell nuclei. In the following, we also use the $a=3$ approximation, when only "bare" NN forces are taken into account, i.e., when $V_{i j k}=0$.

\section{RESULTS}

The main subject of this section will be a careful analysis of the numerical accuracy and convergence properties of the results. The cluster approximation induces a sizeable $\Omega$ dependence, which is driven by the mismatch of the long range behavior of the cluster states and the wave function for the nucleus. The former ones being confined 
by a HO behave like Gaussians, whereas the latter ones decay exponentially for large distances. Because renormalization of the operators is generally weak for the large distances [57, this cannot be corrected by the renormalization of the interaction, but needs to be taken care of by an optimization of the $\Omega$ for the problem and by a rather large model space, which includes enough basis states that the tail is also well approximated. Therefore, to get reliable results, calculations for different model spaces have to be performed for a range of $\Omega$ 's to identify the optimal choice [58].

The model space size is characterized by the maximal number $N$ of $\mathrm{HO}$ excitations included. The lowest ${ }^{7} \mathrm{Li} \mathrm{HO}$ configuration has four nucleons in the $n=0$ HO states and three in the $n=1$ shell. Therefore, the minimal $\mathrm{HO}$ energy is $\mathcal{N}_{\text {min }}=3$. The $A$-body calculations include states up to $\mathcal{N}_{\text {max }}=\mathcal{N}_{\text {min }}+N$ [69]. In this work, we will perform calculations up to $N=6$ including configurations up to $\mathcal{N}_{\max }=9$. The calculations were performed in a basis of single particle Slater-deterimants ( $m$-scheme) using the Many-FermionDynamics code [59, 60]. Since we employ HO single particle states and all Slater determinants up to $\mathcal{N}_{\max }$ are included in the model space, the center of mass part can be exactly separated at the end. For $N=6$, the P-space dimension was 663527 requiring approximately $3 \cdot 10^{9}$ matrix elements for the $A$-body Hamiltonian. The effective interactions are based on $a=3$ clusters. For each model space, we include three-body states up to the maximal excitation of three particles in the $A$-body configurations. For our largest calculation, these are three-body states up to $\mathcal{N}_{3}=9$ and, for the smaller calculations, this number decreases correspondingly. This implies that the effective interactions are different for each model space size. The results for smaller model spaces do not possess upper bound character.

To solve the cluster equation, we use the Lanczosscheme. The usage of a Jacobi basis for this problem guarantees the translational invariance of the effective interaction. This is also mandatory to exclude unphysical center of mass excitations from the model space, which would also complicate the selection of the proper states $|n\rangle$ for the definition of the $\omega$ operator. The cluster equations are solved using the "bare" interactions for Q-spaces up to $\mathcal{N}_{3}=48$ for three-body angular momenta up to $J_{3}=\frac{7}{2}$ and $\mathcal{N}_{3}=40$ for $J_{3}=\frac{9}{2}$ to $\frac{21}{2}$. The largest dimension for the $\mathcal{N}_{3}=9$ P-space is 157 for $J_{3}^{\pi}=\frac{5}{2}^{-}$ and three-body isospin $T_{3}=\frac{1}{2}$ making the same amount of bound state solutions necessary in this channel. Altogether, for all channels between $J_{3}=\frac{1}{2}$ and $J_{3}=\frac{21}{2}$, we required 2352 states. Convergences for the cluster states has been checked using their binding energies. We take the charge dependence of the $\mathrm{NN}$ interaction into account by averaging the $\mathrm{NN}$ isospin $t=1$ interactions according to the prescription given in 61. This averaging was performed for total isospin $T=1 / 2{ }^{7} \mathrm{Li}$ states. All states we will discuss here are $T=1 / 2$ states. The Coulomb interaction is not included into the calculation of the effective

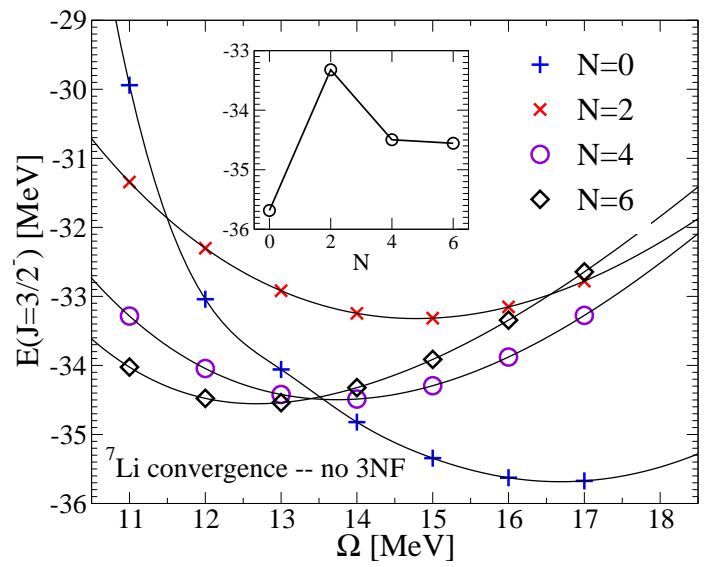

Figure 3: (Color online) $\Omega$ dependence of the binding energy for the ${ }^{7} \mathrm{Li} J^{\pi}=\frac{3}{2}^{-}$ground state using the NN interaction only. Results for $N=0$ to $N=6$ model spaces are shown.

interaction, but is taken into account as an additional "bare" force during the solution of the $A$-body problem. This does not change any results, but will facilitate calculations for $T=3 / 2$ states later, which can now be well approximated using the same effective interactions.

In Figs. 3, 4 and [5] we show the $\Omega$ dependence of the ground state binding energy for ${ }^{7} \mathrm{Li}$ with the IdahoN3LO interaction only and the combinations with 3NF-A and 3NF-B, respectively. The binding energy exhibits a distinct minimum for all model spaces and interactions shown. The position of this minimum shifts to smaller $\Omega$ for larger model spaces. This shift becomes smaller for the larger spaces. Also, the position depends on the interaction model. This behavior has been observed for all realistic interactions and makes the extraction of binding energy results more difficult than for the simpler Minnesota type of interactions. Ref. [58] investigates this issue in detail. There, it was shown that taking the binding energy of the minimum for different model spaces results in a clear pattern of convergence. It was also shown that the results obtained in this way are consistent with results based on the "bare" interaction. Therefore, we also follow this procedure. The inserts in the figures show the convergence of the binding energy based on this idea for model spaces with $N=0,2,4$ and 6 . Very clearly we observe convergence for Idaho-N3LO without $3 \mathrm{NF}$ and with $3 \mathrm{NF}-\mathrm{A}$. For $3 \mathrm{NF}-\mathrm{B}$, the changes in the step $N=4$ to $N=6$ are somewhat larger. They are, however, small enough to justify that the results are accurate to the order of the few hundred keV's or one MeV. This will be sufficient for a discussion of the quality of chiral interactions later.

The results for the excitation energies are shown in Figs. [6 to 8 Fortunately, the $\Omega$ dependence is rather mild and the different excitation energies are very similar for the $N=2,4$ and 6 model spaces. The vertical lines indicate the $\Omega$ of the minima for $N=6$ in Figs. 3 to [5. Our final extraction for the excitation energies is the 


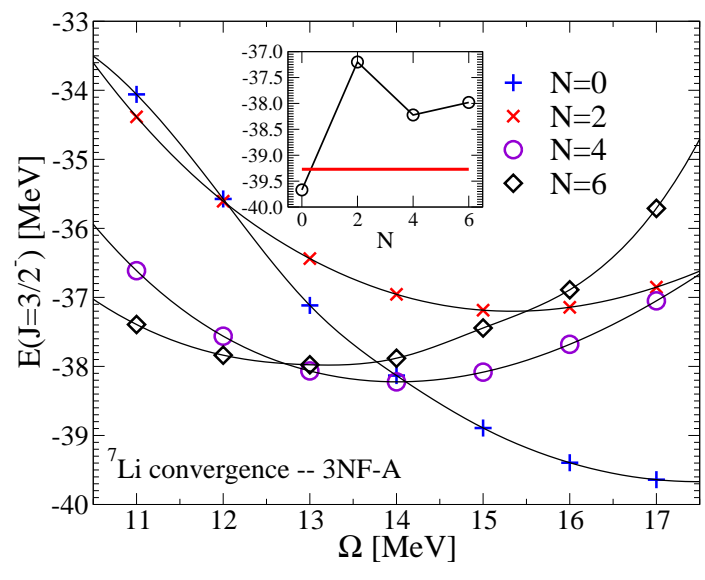

Figure 4: (Color online) $\Omega$ dependence of the binding energy for the ${ }^{7} \mathrm{Li} J^{\pi}=\frac{3}{2}^{-}$ground state using the NN interaction combined with 3NF-A. Results for $N=0$ to $N=6$ model spaces are shown.

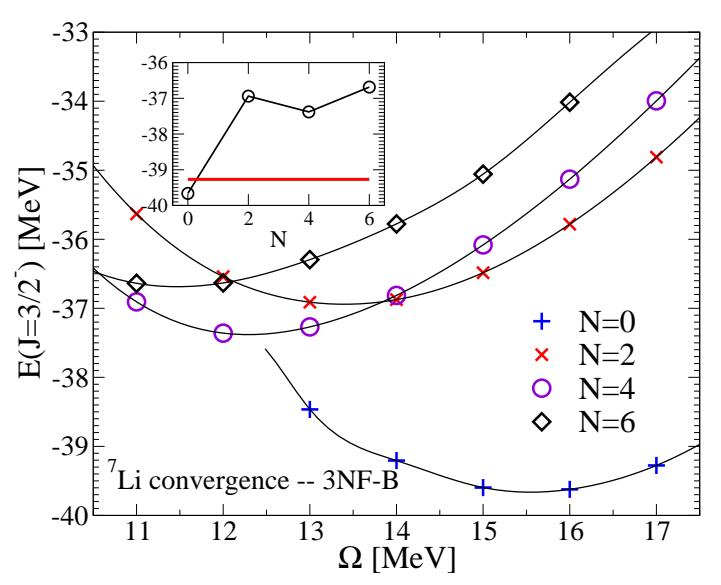

Figure 5: (Color online) $\Omega$ dependence of the binding energy for the ${ }^{7} \mathrm{Li} J^{\pi}=\frac{3}{2}^{-}$ground state using the NN interaction in 3 NF-B. Results for $N=0$ to $N=6$ model spaces are shown.

$N=6$ result at this $\Omega$ value. We also checked the $\Omega$ dependence of the binding energy for the excited $1 / 2^{-}$ state. Since the position of the minima is very similar to the ones for the $3 / 2^{-}$state, we are confident that the excitation energies for the shown $3 / 2^{-}, 1 / 2^{-}, 7 / 2^{-}$and $5 / 2^{-}$are accurate.

Our results for the binding energy are summarized and compared to other calculations and the experiment in Table III Besides our new results, we show results for phenomenological interactions from GFMC calculations. An earlier NCSM results for AV8' and TM' 2] gave $35.8 \mathrm{MeV}$. It was based only on a single value of $\hbar \Omega$, i.e. $14 \mathrm{MeV}$, and on three-body cluster states up to $\mathcal{N}_{3}=28$. For a more reliable binding energy prediction, a complete study of the $\Omega$ dependence is certainly required as well as an increased number of three-body cluster states. Preliminary results clearly show this trend. Note that we use $\mathcal{N}_{3}=48$ in the present study. Because of this, we

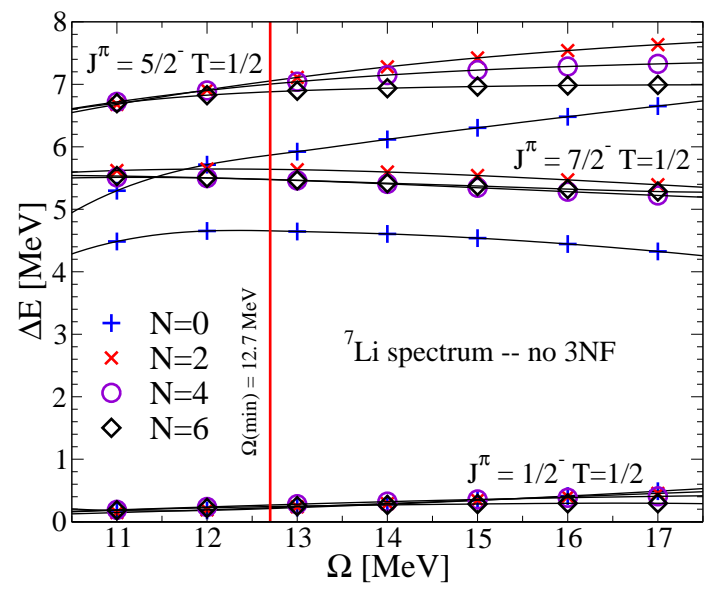

Figure 6: (Color online) $\Omega$ dependence of the excitation energy of the lowest states of ${ }^{7} \mathrm{Li}$ using the NN interaction only. Results for $N=0$ to $N=6$ model spaces are shown.

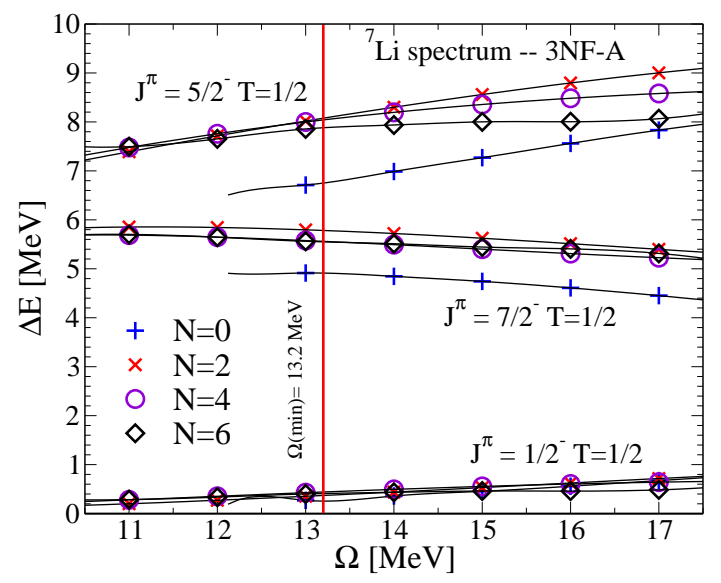

Figure 7: (Color online) $\Omega$ dependence of the excitation energy of the lowest states of ${ }^{7} \mathrm{Li}$ using the NN interaction combined with $3 \mathrm{NF}-\mathrm{A}$. Results for $N=0$ to $N=6$ model spaces are shown.

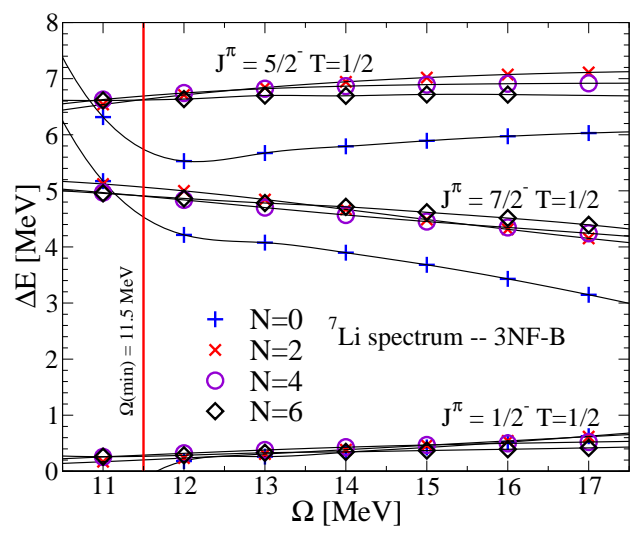

Figure 8: (Color online) $\Omega$ dependence of the excitation energy of the lowest states of ${ }^{7} \mathrm{Li}$ using the NN interaction combined with 3 NF-B. Results for $N=0$ to $N=6$ model spaces are shown. 


\begin{tabular}{l|rr} 
model & $E_{g s}[\mathrm{MeV}]$ & $r[\mathrm{fm}]$ \\
\hline NN only & 34.6 & 2.15 \\
w/ 3NF-A & 38.0 & 2.11 \\
w/ 3NF-B & 36.7 & 2.23 \\
\hline AV8'+TM' [63, 64] & $40.5(1)$ & $2.2(1)$ \\
AV18+Urbana IX [7, 65] & 37.5 & 2.33 \\
AV18+IL2 [7, 65] & 38.9 & 2.25 \\
\hline Expt. [66, 67] & 39.2 & 2.27
\end{tabular}

Table III: Comparison of the ground state binding energy results $E_{g s}$ and the point proton rms radius $r$ for ${ }^{7} \mathrm{Li}$ (for $N=6$ model space and $\Omega$ at the minimum of the energy/ $\Omega$ curve) for chiral interactions and several phenomenological combinations to the experimental value. The experimental rms radius is corrected for the finite size of the proton assuming a proton charge radius of $0.81 \mathrm{fm}^{-1}$.

omit the earlier NCSM result here and compare to the GFMC results.

As expected, ${ }^{7} \mathrm{Li}$ is underbound for the $\mathrm{NN}$ interaction only. Both 3NF's provide more binding. However, in both cases the final binding energy result is still short of the experiment by 1.2 and $2.5 \mathrm{MeV}$, respectively. In view of the general expectation that strong repulsion at short distances is required to avoid a collapse of nuclei, we are encouraged by the slight underbinding. Clearly, we do not observe any sign of overbinding, though neither the NN force nor the $3 \mathrm{NF}$ have a strong repulsive core. Both interactions are very soft, yet the binding energies are reasonable. For phenomenogical, pure $2 \pi$ exchange 3NF's, like the Tucson-Melbourne (TM') 62], overbinding already sets in for ${ }^{7} \mathrm{Li}$. So far, it was believed that only the addition of a repulsive core, like in the UrbanaIX and Illinois models [7, 24], can cure this overbinding problem. Here, we show that the additional structures of chiral 3NF's also prevent overbinding. We note that the description of the binding energy is best for 3NF-A.

The final results for the excitation energies are summarized in Fig. 9 All combinations of the interactions, Idaho-N3LO alone, with 3NF-A or 3NF-B, do predict the right ordering for these states. The splitting of the $3 / 2^{-}$ and $1 / 2^{-}$states is small. The agreement with this experimental splitting seems to be superior for 3NF-A. Because the splitting itself is very small, this might be accidental. More significant deviations of the predictions are observed for the $7 / 2^{-}$and $5 / 2^{-}$states. Both, the position of this multiplet and the splitting is strongly affected by the 3NF's and the agreement with the experimental results is clearly best for $3 \mathrm{NF}-\mathrm{B}$. This is in contradiction to the binding energy, which was best described for parameter set 3NF-A. For a better clarification of this situation, a study of the dependence on the choice of the $c_{i}$ 's is mandatory. This issue is not as simple as it seems at first glance, since the same $c_{i}$ 's enter the $\mathrm{NN}$ interaction. So, in principle, a refitting of the NN interaction would be necessary. We postponed this issue to a later study, where this constraint might be loosened to get more in-

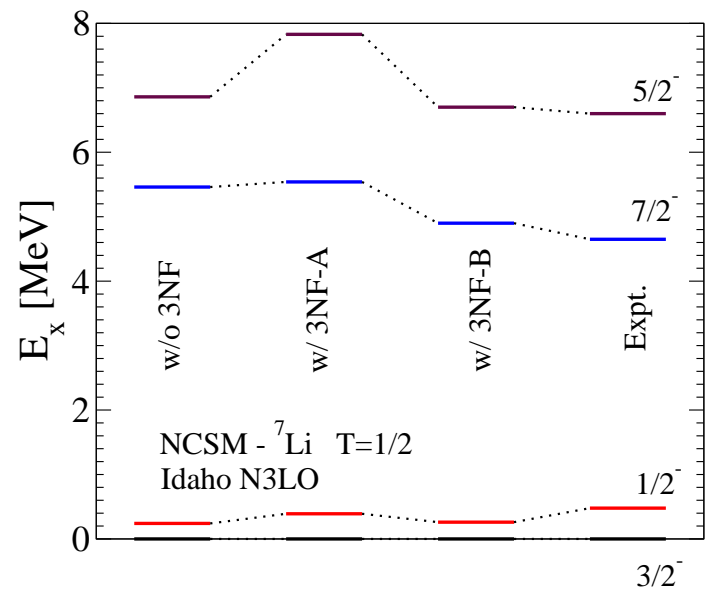

Figure 9: (Color online) Dependence of the excitation energy of the lowest states of ${ }^{7} \mathrm{Li}$ on the interaction. Results with the $\mathrm{NN}$ interaction only and with the 3NF-A and 3NF-B included are compared to the experimental value.

sight into the $c_{i}$ dependence. Finally, we note that part of the deviations for the binding energy might be attributed to our numerically uncertainty. Therefore, the results for ${ }^{7} \mathrm{Li}$ favor the parameter set $3 \mathrm{NF}-\mathrm{B}$, because of its superior description of the spectrum, but further calculations will be required before we can conclude that this result also holds for heavier nuclei.

As we discussed before, we consider one main result of this first calculation to be that the very soft chiral interactions do not predict a collapse of nuclei with increasing number of nucleons. Of course, this also needs to be confirmed for the densities. Therefore, we also show results for the point proton radii for the $3 / 2^{-}$state in Figs. 10 to 12 For this quantity, we found an interesting dependence on $\Omega$ and $N$. For all model spaces, the radii decrease with increasing $\Omega$. This exactly reflects the behavior of the basis HO states. Obviously, there is no correlation to the binding energy result. It is comforting to note that the slope of this dependence decreases with increasing $N$. Also, we observed the smallest dependence on $N$ for the $\Omega$ values around the minima of the binding energy curves, which are again indicated by vertical lines. Therefore, we have good indications that the best extraction of the radii are obtained for exactly these $\Omega$ values for the largest possible model spaces.

Table III also shows the radii obtained in this way and compares them to the experimental values and other calculations. We obtain very realistic results for the chiral interactions, which are comparable to the phenomenological models Urbana-IX, Illinois and TM'. We do not find any indication that soft, chiral interactions fail to saturate nuclear systems with a realistic binding energy and density. We note that for 3NF-B we even observe an increase of the radius though it provides additional attraction. 


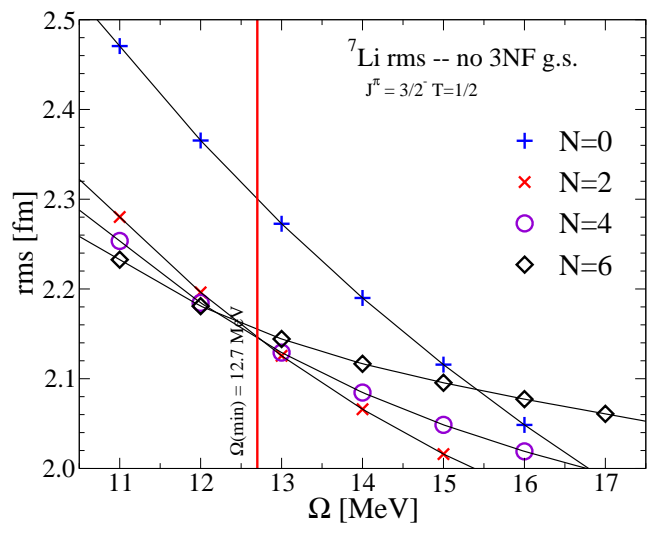

Figure 10: (Color online) $\Omega$ dependence of the point proton rms radius of the ground state of ${ }^{7} \mathrm{Li}$ using the $\mathrm{NN}$ interaction only. Results for $N=0$ to $N=6$ model spaces are shown.

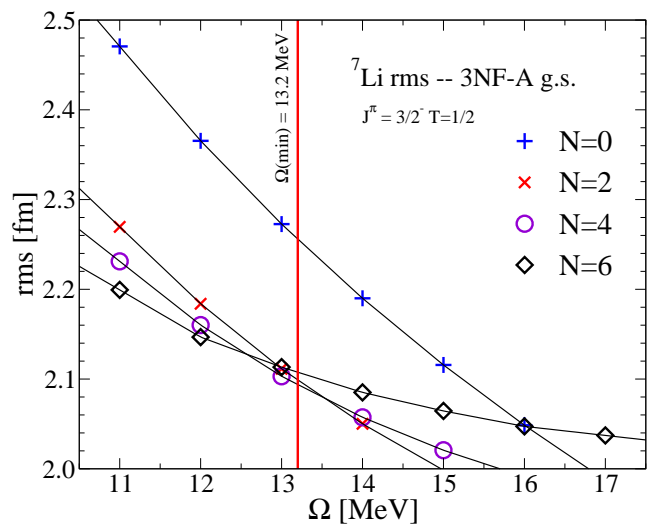

Figure 11: (Color online) $\Omega$ dependence of the point proton rms radius of the ground state of ${ }^{7} \mathrm{Li}$ using the NN interaction combined with 3NF-A. Results for $N=0$ to $N=6$ model spaces are shown.

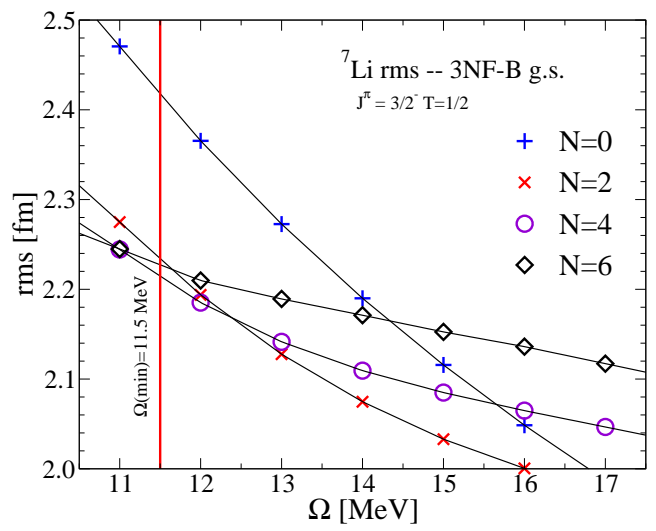

Figure 12: (Color online) $\Omega$ dependence of the point proton rms radius of the ground state of ${ }^{7} \mathrm{Li}$ using the NN interaction combined with 3NF-B. Results for $N=0$ to $N=6$ model spaces are shown.

\section{CONCLUSIONS}

We have presented the first microscopic calculation of the low lying ${ }^{7} \mathrm{Li}$ states based on a full chiral interaction, including $\mathrm{NN}$ and $3 \mathrm{~N}$ forces. Our results are based on the NCSM. We showed that predictions are possible with an accuracy that allows one to discuss the basic properties of the nuclear force. The results confirm that binding energies and spectra depend on the structure of the 3NF's. This means that the results for different sets of parameters for the $3 \mathrm{NF}$ are different though both models describe the ${ }^{3} \mathrm{H}$ and ${ }^{4} \mathrm{He}$ nuclei with the same accuracy. In this first application, we restricted ourselves to changes in the strength of the short range, or short-range long range pieces (D- and E-terms) of the $3 \mathrm{NF}$, which are not related to the NN interaction at the order $Q^{3}$. With this constraint, we have already obtained reasonable results for both, the binding energies and the spectra, but we could not describe both simultaneously within the accuracy, which we expect from our many-body method.

On the other hand, we know that the strength of $2 \pi$ exchange in the $\mathrm{NN}$ and $3 \mathrm{~N}$ interactions is not well determined yet. Therefore, it is necessary to establish a measure for the dependence of the ${ }^{7} \mathrm{Li}$ and other $p$-shell results on this strength. Since, in principle, this can only be done by changing the $\mathrm{NN}$ and $3 \mathrm{~N}$ forces, it is not a straightforward and simple task and, probably, for first estimates, one will need to loosen the strict consistency of $\mathrm{NN}$ and $3 \mathrm{~N}$ forces somewhat. This will be the subject of a forthcoming study.

Here, we were mainly interested to establish that soft chiral interactions result in realistic binding energies and densities. We therefore also investigated the point proton radii and described how these can be extracted from NCSM wave functions. The results support that soft, low momentum interactions predict reasonable binding energies and densities. In the future, this conjecture needs to be confirmed for heavier systems. In this respect, a study of ${ }^{10} \mathrm{~B}$ is a high priority. Results employing the TM' $3 \mathrm{NF}$ and the AV8' $\mathrm{NN}$ force indicate an increasing overbinding going from $A=7$ to $A=10$ [63]. It needs to be confirmed that the chiral interactions can again avoid overbinding. At the same time, ${ }^{10} \mathrm{~B}$ has proven to be very sensitive to the spin-orbit interactions including the 3NF's. Usual NN interactions, like Idaho-N3LO and AV18 2, 7, 58], predict a wrong ordering of the $3^{+}$ground and $1^{+}$excited state. The Illinois type of 3NF's correctly change this ordering and also the TM' 3NF affects this positively, in contrast to the Urbana IX 3NF. Therefore, the predictions of the complete chiral interaction with the $3 \mathrm{NF}$ are especially interesting for this nucleus. But also for this system, the dependence on the $c_{i}$ 's of the $2 \pi$ exchange should be studied. 


\section{Acknowledgments}

We acknowledge partial support by the US DOE under Grants No. DE-FC02-01ER41187, DE-FG0200ER41132, and DE-FG-02-87ER-40371, and by NSF Grant Nos. PHY0070858 and PHY0244389. This work was partly performed under the auspices of the U.S. Department of Energy by the University of California, Lawrence Livermore National Laboratory under Contract No.W-7405-Eng-48. Support from the LDRD Contract No. 04-ERD-058, and from the U.S. Department of Energy, Office of Science, (Work Proposal Number SCW0498) is acknowledged. A.N. and B.R.B were partially supported by the NATO Collaborative Linkage Grant 2004/2005. The numerical calculations have been performed on Seaborg at the National Energy Research Scientific Computing Center (NERSC), Berkeley.

\section{Appendix A: EXPLICIT EXPRESSIONS FOR THE CHIRAL 3NF'S}

In this appendix, we would like to summarize the explicit form of the chiral $3 \mathrm{NF}$. The explicit expressions were first derived in [4]. The $3 \mathrm{NF}$ consists of terms with the three different topologies. The first one is the usual $2 \pi$ exchange. The operator structure is identical to the well known Tucson-Melbourne interaction [25]. Then there are two new topologies, which contain also contact interactions (see e.g. 18]). In the original work, five independent terms with such structures are derived. In [18], it was realized that the number of independent terms is smaller once the antisymmetry of the $3 \mathrm{~N}$ states is taken into account. Then only one term of the $1 \pi$ exchange type (D-term) and one term of the pure contact type (E-term) are independent.
Following the notation of [68], the $2 \pi$ exchange part reads

$$
V_{i j k}^{(k) ; 2 \pi}=\sum_{i \neq j \neq k} \frac{1}{2}\left(\frac{g_{A}}{2 F_{\pi}}\right)^{2} \frac{\left(\vec{\sigma}_{i} \cdot \vec{q}_{i}\right)\left(\vec{\sigma}_{j} \cdot \vec{q}_{j}\right)}{\left(\vec{q}_{i}^{2}+m_{\pi}^{2}\right)\left(\vec{q}_{j}^{2}+m_{\pi}^{2}\right)} F_{i j k}^{\alpha \beta} \tau_{i}^{\alpha} \tau_{j}^{\beta}
$$

where $\vec{q}_{i}$ is the momentum of the pion exchanged between nucleons $i$ and $k$ and

$F_{i j k}^{\alpha \beta}=\delta^{\alpha \beta}\left[-\frac{4 c_{1} m_{\pi}^{2}}{F_{\pi}^{2}}+\frac{2 c_{3}}{F_{\pi}^{2}} \vec{q}_{i} \cdot \vec{q}_{j}\right]+\sum_{\gamma} \frac{c_{4}}{F_{\pi}^{2}} \epsilon^{\alpha \beta \gamma} \tau_{k}^{\gamma} \vec{\sigma}_{k} \cdot\left[\vec{q}_{i} \times \vec{q}_{j}\right]$

The strength of these terms is completely determined by the $c_{i}$ constants discussed in the main text. The two new terms read

$V_{i j k}^{(k) ; 1 \pi}=-\sum_{i \neq j \neq k} \frac{g_{A}}{8 F_{\pi}^{2}} \frac{c_{D}}{F_{\pi}^{2} \Lambda_{\chi}} \frac{\vec{\sigma}_{j} \cdot \vec{q}_{j}}{\vec{q}_{j}^{2}+m_{\pi}^{2}}\left(\tau_{i} \cdot \tau_{j}\right)\left(\vec{\sigma}_{i} \cdot \vec{q}_{j}\right)$

and

$$
V_{i j k}^{(k) ; \text { cont }}=\frac{1}{2} \sum_{i \neq j \neq k} \frac{c_{E}}{F_{\pi}^{4} \Lambda_{\chi}}\left(\tau_{j} \cdot \tau_{k}\right)
$$

In these terms two new strength constants $c_{D}$ and $c_{E}$ appear. The values, which we determine as described in the main text from the binding energies of ${ }^{3} \mathrm{H}$ and ${ }^{4} \mathrm{He}$, are based on the choice $\Lambda_{\chi}=700 \mathrm{MeV}$. For the axial vector coupling constant, we use $g_{A}=1.29$. $F_{\pi}=$ $92.4 \mathrm{MeV}$ is the weak pion decay constant and $m_{\pi}=$ 138.03 MeV the averaged pion mass. As outlined in [18], we regularize the $3 \mathrm{NF}$ using a cutoff. Throughout this paper, we fix the cutoff to $\Lambda=500 \mathrm{MeV}$.
[1] S. C. Pieper and R. B. Wiringa, Ann. Rev. Nucl. Part. Sci. 51, 53 (2001).

[2] P. Navrátil and W. E. Ormand, Phys. Rev. C 68, 034305 (2003).

[3] V. G. J. Stoks, R. A. M. Klomp, C. P. F. Terheggen, and J. J. de Swart, Phys. Rev. C 49, 2950 (1994).

[4] R. B. Wiringa, V. G. J. Stoks, and R. Schiavilla, Phys. Rev. C 51, 38 (1995).

[5] R. Machleidt, F. Sammarruca, and Y. Song, Phys. Rev. C 53, R1483 (1996).

[6] R. Machleidt, Phys. Rev. C 63, 024001 (2001).

[7] S. C. Pieper, V. R. Pandharipande, R. B. Wiringa, and J. Carlson, Phys. Rev. C 64, 014001 (2001).

[8] T.-S. Park, L. E. Marcucci, R. Schiavilla, M. Viviani, A. Kievsky, S. Rosati, K. Kubodera, D.-P. Min, and M. Rho, Phys. Rev. C 67, 055206 (2003).

[9] V. Bernard, N. Kaiser, and U.-G. Meißner, Int. J. of Mod. Phys. E 4, 193 (1995).

[10] U. van Kolck, Prog. Part. Nucl. Phys. 43, 337 (1999).

[11] P. F. Bedaque and U. van Kolck, Ann. Rev. Nucl. Part.
Sci. 52, 339 (2002).

[12] E. Epelbaum, nucl-th/0509032.

[13] H. Witała, W. Glöckle, J. Golak, A. Nogga, H. Kamada, R. Skibiński, and J. Kuroś-Żołnierczuk, Phys. Rev. C 63, 024007 (2001).

[14] R. Bieber, W. Glöckle, J. Golak, M. N. Harakeh, D. Hüber, H. Huisman, N. Kalantar-Nayestanaki, H. Kamada, J. G. Messchendorp, A. Nogga, et al., Phys. Rev. Lett. 84, 606 (2000).

[15] H. Sakai, K. Sekiguchi, H. Witała, W. Glöckle, M. Hatano, H. Kamada, H. Kato, Y. Maeda, A. Nogga, T. T. Ohnishi, et al., Phys. Rev. Lett. 84, 5288 (2000).

[16] R. V. Cadman, J. Brack, W. J. Cummings, J. A. Fedchak, B. D. Fox, H. Gao, W. Glöckle, J. Golak, C. Grosshauser, R. J. Holt, et al., Phys. Rev. Lett. 86, 967 (2001).

[17] K. Ermisch, A. M. van den Berg, R. Bieber, W. Glöckle, J. Golak, M. Hagemann, V. M. Hannen, M. N. Harakeh, M. A. de Huu, N. Kalantar-Nayestanaki, et al., Phys. Rev. Lett. 86, 5862 (2001).

[18] E. Epelbaum, A. Nogga, W. Glöckle, H. Kamada, U.-G. 
Meißner, and H. Witała, Phys. Rev. C 66, 064001 (2002).

[19] W. Polyzou and W. Glöckle, Few-Body Systems 9, 97 (1990).

[20] P. Navrátil, J. P. Vary and B. R. Barrett, Phys. Rev. Lett. 84, 5728 (2000).

[21] P. Navrátil, J. P. Vary and B. R. Barrett, Phys. Rev. C 62, 054311 (2000).

[22] A. Nogga, H. Kamada, W. Glöckle, and B. R. Barrett, Phys. Rev. C 65, 054003 (2002).

[23] P. Navrátil and B. R. Barrett, Phys. Rev. C 57, 3119 (1998).

[24] B. S. Pudliner, V. R. Pandharipande, J. Carlson, S. C. Pieper, and R. B. Wiringa, Phys. Rev. C 56, 1720 (1997).

[25] S. A. Coon, M. D. Scadron, P. C. McNamee, B. R. Barrett, D. W. E. Blatt, and B. H. J. McKellar, Nucl. Phys. A317, 242 (1979).

[26] E. Braaten and H. W. Hammer, cond-mat/0410417.

[27] L. Platter, H. W. Hammer, and U.-G. Meißner, Phys. Lett. B 607, 254 (2005).

[28] S. Weinberg, Physica A96, 327 (1979).

[29] N. Fettes, U.-G. Meißner, and S. Steininger, Phys. Lett. B451, 233 (1999).

[30] U. van Kolck, J. L. Friar, and T. Goldman, Phys. Lett. B371, 169 (1996).

[31] S. Weinberg, Phys. Lett. B251, 288 (1990).

[32] S. Weinberg, Nucl. Phys. B363, 3 (1991).

[33] S. Weinberg, Phys. Lett. B295, 114 (1992).

[34] C. Ordóñez, L. Ray, and U. van Kolck, Phys. Rev. C 53, 2086 (1996).

[35] E. Epelbaum, W. Glöckle, and U.-G. Meißner, Nucl. Phys. A671, 295 (2000).

[36] D. R. Entem and R. Machleidt, Phys. Lett. B524, 93 (2002).

[37] D. R. Entem and R. Machleidt, Phys. Rev. C 68, 041001(R) (2003).

[38] E. Epelbaum, W. Glöckle, and U.-G. Meißner, Nucl. Phys. A747, 362 (2005).

[39] D. B. Kaplan, M. J. Savage, and M. B. Wise, Phys. Lett. B424, 390 (1998).

[40] S. Fleming, T. Mehen, and I. W. Stewart, Nucl. Phys. A677, 313 (2000).

[41] P. Büttiker and U.-G. Meißner, Nucl. Phys. A668, 97 (2000).

[42] M. C. M. Rentmeester, R. G. E. Timmermans, and J. J. de Swart, Phys. Rev. C 67, 044001 (2003).

[43] V. G. J. Klomp, R. A. M. Stoks and J. J. de Swart, Phys. Rev. C 44, R1258 (1991).

[44] U. van Kolck, Phys. Rev. C 49, 2932 (1994).
[45] N. Fettes, U.-G. Meißner, and S. Steininger, Nucl. Phys. A640, 199 (1998).

[46] K. Suzuki and S. Y. Lee, Progr. of Theor. Phys. 64, 2091 (1980).

[47] K. Suzuki, Progr. of Theor. Phys. 68, 246 (1982).

[48] S. Okubo, Progr. Theor. Phys. 12, 603 (1954).

[49] E. Epelbaoum, W. Glöckle, and U.-G. Meißner, Nucl. Phys. A637, 107 (1998).

[50] E. Epelbaoum, W. Glöckle, A. Krüger, and U.-G. Meißner, Nucl. Phys. A645, 413 (1998).

[51] S. K. Bogner, T. T. S. Kuo, and A. Schwenk, Phys. Rep. 386, 1 (2003).

[52] N. Barnea, W. Leidemann, and G. Orlandini, Phys. Rev. C 61, 054001 (2000).

[53] N. Barnea, W. Leidemann, and G. Orlandini, Nucl. Phys. A693, 565 (2001).

[54] H. J. Lipkin, Phys. Rev. 109, 2071 (1958).

[55] P. Navrátil and B. R. Barrett, Phys. Rev. C 54, 2986 (1996).

[56] C. P. Viazminsky and J. P. Vary, J. of Math. Phys. 42, 2055 (2001).

[57] I. Stetcu, B. R. Barrett, P. Navrátil, and J. P. Vary, Phys. Rev. C 71, 044325 (2005).

[58] P. Navrátil and E. Caurier, Phys. Rev. C 69, 014311 (2004).

[59] J. P. Vary, The many-fermion-dynamics shell-model code (1992), (unpublished).

[60] J. P. Vary and D. C. Zheng, The many-fermion-dynamics shell-model code (1994), (unpublished).

[61] G. P. Kamuntavičius, P. Navrátil, B. R. Barrett, G. Sapragonaite, and R. K. Kalinauskas, Phys. Rev. C 60, 044304 (1999).

[62] S. A. Coon and H. K. Han, Few Body Systems 30, 131 (2001).

[63] S. C. Pieper, Nucl. Phys. A751, 516c (2005).

[64] S. C. Pieper, private communication.

[65] S. C. Pieper, R. B. Wiringa, and J. Carlson, Phys. Rev. C 70, 054325 (2004).

[66] D. R. Tilley, C. M. Cheves, J. L. Godwin, G. M. Hale, H. M. Hofmann, J. H. Kelley, C. G. Shen, and H. R. Weller, Nucl. Phys. A 708, 3 (2002).

[67] H. de Vries, C. W. de Jager, and C. de Vries, At. Data Nucl. Data Tables 36, 495 (1987).

[68] J. L. Friar, D. Hüber, and U. van Kolck, Phys. Rev. C 59, 53 (1999).

[69] Note that in previous works, what we call " $N$ " here, was labeled " $N_{\max }$ ". 